\title{
ARTICLE Efficacy, tolerability, and cognitive effects of deep transcranial magnetic stimulation for late-life depression: a prospective randomized controlled trial
}

Tyler S. Kaster ${ }^{1,2}$, Zafiris J. Daskalakis ${ }^{1,2,3}$, Yoshihiro Noda ${ }^{4}$, Yuliya Knyahnytska ${ }^{1,2}$, Jonathan Downar $\mathbb{( D}^{2,5}$, Tarek K. Rajji ${ }^{2,3,6}$, Yechiel Levkovitz ${ }^{7}$, Abraham Zangen ${ }^{8}$, Meryl A. Butters ${ }^{9}$, Benoit H. Mulsant ${ }^{2,3,6}$ and Daniel M. Blumberger ${ }^{1,2,3,6}$

Late-life depression (LLD) is a growing worldwide problem due to demographic changes, with limited treatment options due to high rates of pharmacotherapy adverse effects, accessibility of psychotherapy, and tolerability of electroconvulsive therapy. Novel neuromodulation techniques, such as repetitive transcranial magnetic stimulation (rTMS), may overcome these limitations. The objective of this study is to determine the efficacy, tolerability, and cognitive effects of high-dose deep rTMS in LLD. In this study we randomized older adults between 60 and 85 years old with major depressive disorder (MDD) to sham or active deep rTMS (H1 coil, 6012 pulses, $18 \mathrm{~Hz}, 120 \%$ of resting motor threshold) delivered over the dorsolateral and ventrolateral prefrontal cortex 5 days per week over 4 weeks. Our primary outcome was remission of depression in an intention-to-treat analysis. We also assessed change in cognitive functioning with rTMS treatment and tolerability based on adverse effects. Fifty-two participants were randomized to active $(n=25)$ or sham H1 coil $(n=27)$. Remission rate was significantly higher with active than sham rTMS $(40.0 \%$ vs $14.8 \%)$ with a number needed to treat of 4.0 (95\% Cl: $2.1-56.5)$. There was no change on any measure of executive function and no serious adverse events. Adverse effect profiles were similar between active and sham rTMS, except for reports of pain being significantly more common in the active condition ( $16.0 \%$ vs $0 \%)$. High-dose deep rTMS appears to be safe, well tolerated, and efficacious in the treatment of LLD.

Neuropsychopharmacology (2018) 43:2231-2238; https://doi.org/10.1038/s41386-018-0121-x

\section{INTRODUCTION}

Depression has recently become the leading cause of illness burden worldwide [1] and occurs in $7 \%$ of adults age 60 years and older [2]. With worldwide demographic changes, the burden of late-life depression (LLD) is rapidly increasing [2]. When treated with antidepressant medication, many older adults experience adverse effects, drug-drug interactions, or do not respond to treatment $[3,4]$. Non-pharmacotherapy alternatives for LLD are limited due to issues of accessibility for evidence-based psychotherapy [5] and tolerability for electroconvulsive therapy (ECT) [6]. Therefore, the development and assessment of new nonpharmacological treatments is needed.

Over the past decade, repetitive transcranial magnetic stimulation (rTMS) has demonstrated effectiveness and tolerability for the treatment of depression in younger adults [7]. In a recent network meta-analysis, active rTMS was associated with higher odds of response than sham rTMS [8]. However, in the few studies evaluating rTMS for LLD, older age has been a predictor of nonresponse to rTMS [9]. The reasons for this finding remain unclear but it has been suggested that age-associated brain atrophy and inadequate rTMS dosing have contributed to this poor response.
First, previous studies have shown that age-related prefrontal cortical atrophy increases the distance between scalp and cortex, necessitating higher stimulation intensities [10-12]. This increased scalp-cortex distance may also impede conventional rTMS coils from achieving adequate cortical penetration necessary for therapeutic efficacy. Therefore, effective treatment of LLD with rTMS may require coil designs that provide sufficient cortical penetration. This may be possible with an $\mathrm{H} 1$ coil, which has been designed to stimulate deeper and larger brain volumes [13-17]. This coil design has been shown to be safe and efficacious in open-label trials of younger adults with depression [16, 18, 19], and in a recent multicenter sham-controlled randomized trial [20]. This latter trial included patients with major depressive disorder (MDD) up to age 68 years, and the mean age of participants was 46. The $\mathrm{H} 1$ coil is generally well tolerated, though, similar to conventional rTMS coils, there have been reports of accidental seizure induction [19, 21-24]. Second, it has been hypothesized that the treatment of LLD requires stimulation intensities that can overcome prefrontal atrophy [25]. In addition, early rTMS studies that have included older adults likely delivered too few pulses [26]. Indeed, pivotal rTMS trials delivered 3000 pulses daily had

\footnotetext{
${ }^{1}$ Temerty Centre for Therapeutic Brain Intervention, Centre for Addiction and Mental Health, Toronto, ON, Canada; ${ }^{2}$ Department of Psychiatry, University of Toronto, Toronto, ON, Canada; ${ }^{3}$ Campbell Family Mental Health Research Institute, Centre for Addiction and Mental Health, Toronto, ON, Canada; ${ }^{4}$ Department of Neuropsychiatry, Keio University School of Medicine, Tokyo, Japan; ${ }^{5}$ MRI-Guided rTMS Clinic, Toronto Western Hospital, Toronto, ON, Canada; ${ }^{6}$ Geriatric Psychiatry Division, Centre for Addiction and Mental Health, Toronto, ON, Canada; ${ }^{7}$ Be'er-Ya'akov Mental Health Center, Tel Aviv University, Be'er-Ya'akov, Israel; ${ }^{8}$ Department of Life Sciences and the Zlotowsky Neuroscience Center, Ben-Gurion University of the Negev, Be'er Sheva, Israel and ${ }^{9}$ Department of Psychiatry, University of Pittsburgh School of Medicine, Pittsburgh, PA, USA Correspondence: Daniel M. Blumberger (daniel.blumberger@camh.ca)
}

Received: 13 November 2017 Revised: 11 June 2018 Accepted: 12 June 2018

Published online: 18 June 2018 
minimal adverse effects $[27,28]$. There is both neurophysiological and clinical evidence to suggest that increasing the number of daily pulses may increase response rates. First, neurophysiological data suggest that a single session of 6000 pulses of rTMS delivered at $20 \mathrm{~Hz}$ increases cortical inhibition-a marker of treatment response for brain stimulation treatments $[29,30]$-compared to 1200 or 3600 pulses [31). Second, clinical trials using accelerated rTMS treatment protocols that delivered 15 sessions of 1000 pulses of rTMS over 2 days resulted in rapid treatment response [32].

In addition to the efficacy of deep rTMS in LLD, we also sought to determine its impact on cognitive functioning, including executive functioning which is frequently impaired in LLD [33, 34]. Previous studies in younger adults have found that rTMS treatment is associated with improvements in cognitive functioning independent of mood changes [35], and a recent systematic review of the association between rTMS and executive functioning in older adults found the executive function benefits from rTMS were positively related to mood improvement in LLD [36]. To date, however, there are no studies examining the association between deep rTMS and cognitive functioning in LLD.

Therefore, we conducted a prospective two-armed parallel superiority randomized control trial to evaluate the rates of LLD remission using high-dose deep rTMS with an $\mathrm{H} 1$ coil, compared to a sham condition, in older adults with LLD. We also sought to determine the tolerability and impact on cognitive functioning of deep rTMS compared to a sham condition. We hypothesized that, compared to sham treatment, active rTMS would be associated with higher remission rates, similar tolerability, and improvements in cognitive functioning.

\section{METHODS}

\section{Participants}

This was a double-blind, randomized, sham-controlled trial conducted at the Center for Addiction and Mental Health (CAMH), a 530-bed academic psychiatric hospital in Toronto, Canada. The study was approved by the CAMH research ethics board, and all participants provided written informed consent at the time of enrollment into the study. Participants were outpatients between the ages of 60 and 85 years with a diagnosis of MDD confirmed using the Structured Clinical Interview for DSM-IV (SCID) [37]. They met the following additional inclusion criteria: current major depressive episode with a score of $\geq 22$ on the 24-item Hamilton Depression Rating Scale (HDRS-24) [38]; lack of response to at least one adequate or two inadequate antidepressant trials during the current episode, as assessed by the Antidepressant Treatment History Form (ATHF) [39]; and receiving stable dosages of psychotropic medications for at least 4 weeks prior to screening. The exclusion criteria were: substance dependence/abuse less than 3 months preceding study entry; unstable medical/neurologic illness; acute suicidality; SCID-IV diagnosis of bipolar I or II disorder; primary psychotic disorder; psychotic symptoms in current episode; primary diagnosis of obsessive-compulsive, post-traumatic stress, anxiety, or personality disorder; probable dementia diagnosis based on a Mini Mental Status Exam (MMSE) score of $<26$ and clinical evidence of dementia; rTMS contraindication (i.e., history of seizures; intracranial implant); failed ECT trial during current episode; previous rTMS treatment; receiving bupropion $>300 \mathrm{mg} /$ day due to dose-dependent increased risk of seizures [40]; receiving lorazepam $>2 \mathrm{mg} /$ day or any anticonvulsant due to reduced cortical excitability which may interfere with rTMS efficacy [41, 42]; or significant laboratory abnormalities.

\section{Study design \\ Participants were randomized to active rTMS or sham rTMS, administered 5 days per week for a total of 20 treatments over} 4 weeks, and continued their psychotropic medications unchanged for the trial duration. Participants who achieved remission by the end of week 4 (defined as both HDRS- $24 \leq 10$ and $\geq 60 \%$ reduction from baseline on 2 consecutive weeks) then continued with twice weekly rTMS for 2 weeks ( 4 additional treatments) to improve the likelihood of a durable remission. Participants who did not meet criteria for remission exited the study at 4 weeks. Participants were withdrawn if: HDRS-24 increased from baseline $>25 \%$ on two consecutive assessments, they developed significant suicidal ideation, or attempted suicide. The target sample size was 80 to ensure statistical power of 0.8 based on a power analysis assuming a type I error rate of 0.05 , sham condition remission rate of $10 \%$, active treatment group remission rate of $36 \%$, and 1:1 allocation between treatment groups. These remission rates were based on previous studies using the same deep rTMS device [20,43].

\section{Randomization and blinding}

Participants were randomized (1:1) using a permuted block method with a random number generator prepared by an independent study consultant. Blocks were of fixed size and study personnel were blinded to randomization block size. Participants were stratified by treatment-resistance (ATHF $\geq 3$ or $<3$ ), and were blinded to treatment condition. Study blind was assessed after the first treatment when participants were asked whether they had received active or sham stimulation. Clinical evaluators and study investigators were also blinded to treatment condition. To ensure allocation concealment, randomization was managed by an independent assistant who assigned a unique participant number and condition code for each participant. The unique participant number and condition code matched a preprogrammed treatment card. The treating technician then inserted the participant's pre-programmed card to activate the active or sham mode. This ensured that operators were also blinded to the randomized condition.

\section{rTMS technique}

We administered rTMS using a Brainsway deep rTMS system with the $\mathrm{H} 1$ coil device (Brainsway Ltd, Jerusalem, Israel). Intensity was derived using resting motor threshold (RMT) obtained before treatment according to previously published methods [44]. The first six participants (five in active and one in sham group) received treatment with an $\mathrm{H} 1 \mathrm{~L}$ helmet coil which stimulates entirely over the left dorsolateral prefrontal cortex. However, this coil was found to be poorly tolerated (described below). As such, the protocol was revised and the $\mathrm{H} 1$ coil was used for all subsequent participants. The participants who received treatment with the $\mathrm{H} 1 \mathrm{~L}$ coil are not included in subsequent analyses due to substantial differences in the electric field properties of this coil [43]. Three of these six participants did not complete the intervention, two in the active and one in the sham condition. One of the participants who did not complete the intervention was in the active $\mathrm{H} 1 \mathrm{~L}$ condition and experienced a seizure 1 day after the 10th session; the other participant in the active $\mathrm{H} 1 \mathrm{~L}$ condition who dropped out was unable to tolerate the stimulus due to pain at the site of stimulation. The participant in the sham H1L condition who dropped out was also unable to tolerate the stimulus due to pain at the stimulation site. Other adverse effects experienced in the active $\mathrm{H} 1 \mathrm{~L}$ condition included: headache $(n=1)$, pain $(n=$ $1)$, and nasopharyngitis ( $n=1)$.

All subsequent rTMS sessions were delivered with the $\mathrm{H} 1$ coil targeting the dorsolateral and ventrolateral prefrontal cortex bilaterally, with greater intensity and penetration of the left hemisphere $[14,43]$, and performed at $120 \%$ of the RMT, similar to previous studies of depression [43]. The active rTMS group received the following standardized dose of rTMS: $18 \mathrm{~Hz}$, at $120 \%$ RMT, $2 \mathrm{~s}$ pulse train, $20 \mathrm{~s}$ inter-train interval, 167 trains, for a total of 6012 pulses per session over $61 \mathrm{~min}$. The control group 


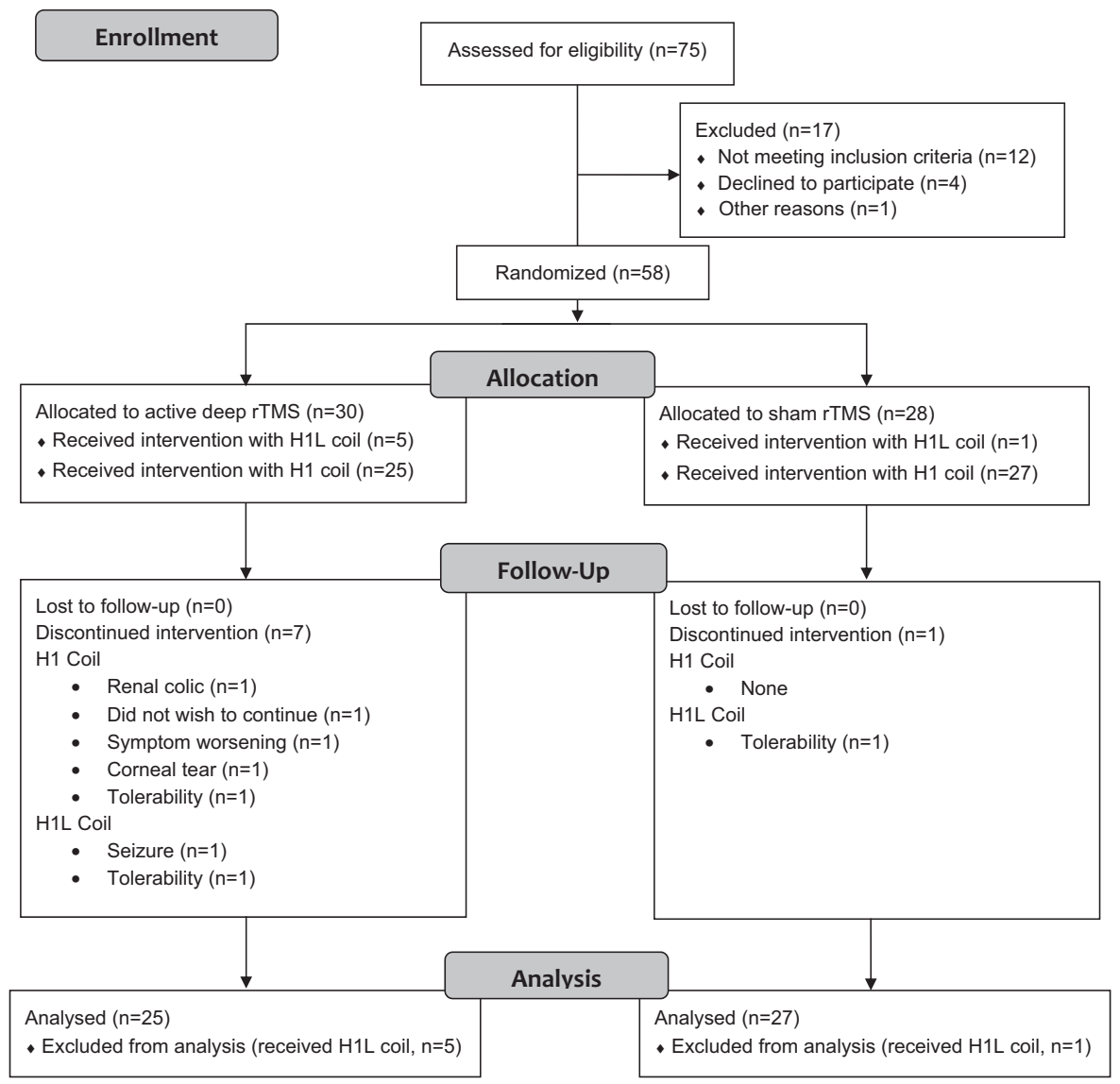

Fig. 1 CONSORT diagram depicting flow of participants through study. rTMS repetitive transcranial magnetic stimulation

received a sham intervention with identical parameters, device, and helmet. However, when sham mode was initiated, the active $\mathrm{H} 1$ coil was disabled, and a second coil (sham $\mathrm{H} 1$ coil) located within the treatment helmet but far above the participant's scalp was activated. This sham $\mathrm{H} 1$ coil delivered a similar tactile and auditory sensation as the active $\mathrm{H} 1$ coil, but the electric field was insufficient to induce neuronal activation.

Assessments and outcomes

The following clinical dimensions were assessed at baseline: depressive symptoms using HDRS-24; suicidal ideation using the Scale for Suicidal Ideation (SSI) [45]; health-related quality of life (HRQoL) using the 36-Item Short Form Survey (SF-36) [46]; anxiety using the Brief Symptom Inventory anxiety subscale (BSI) [47]; and cognitive function using the Repeatable Battery for the Assessment of Neuropsychological Status (RBANS) [48]; and two subscales from the Delis-Kaplan Executive Function System (DKEFS): Color Word Interference (DKEFS-CWI) (measuring response inhibition) and Trail Making Test (DKEFS-TMT) (measuring set-shifting) [49]. The following measures were repeated weekly during the intervention: HDRS-24, SSI, and BSI; or at study end: SF-36, RBANS, and DKEFS. Adverse events were recorded by the rTMS operator after every session.

The primary outcome was remission defined as described above. Secondary efficacy outcomes included response rate ( $>50 \%$ reduction in HDRS- 24 relative to baseline on 2 consecutive weeks), and treatment-attributable change in HDRS-24. Other secondary efficacy outcomes included treatment-attributable change in suicidal ideation, anxiety, $\mathrm{HRQOL}$, and executive functioning. Safety and tolerability were assessed by comparing adverse event rates between the two conditions.
Analysis

We compared baseline differences in demographic and clinical characteristics between active and sham rTMS conditions as well as between study dropouts and completers. We assessed group differences in these factors using chi-square analyses or Fisher's exact test, Student's $t$-test, or Wilcoxon rank sum test as appropriate. Success of blinding was assessed using the $\mathrm{K}$ statistic. For study outcomes, analyses were completed according to the intention-to-treat principle, except where indicated otherwise. For our primary outcome we calculated the proportion of participants meeting remission criteria; number needed to treat (NNT) to achieve remission; the probability of remission with active relative to sham rTMS (RP) and $95 \%$ confidence intervals (Cls). We used a linear mixed-effects model to determine treatment-attributable changes in our efficacy and cognitive outcome measures (HDRS-24, SSI, BSI, SF-36, RBANS, and DKEFS) over time and we compared them in the sham and active rTMS conditions. For cognitive assessments using RBANS, to ensure comparability between pre- and post-treatment assessments, we calculated a Z-score based on the particular version's (A or B) normative mean and standard deviation. The model used time, treatment, and treatment by time interaction as fixed effects. Time was considered a categorical variable with five levels for the weekly assessments from baseline to week 4 . Participants entered the model as random effects, which imposes a compound symmetry structure to the errors within each participant. Our focus was on the treatment by time interaction and whether it was significant at $a<0.05$. A significant interaction was interpreted as offering evidence that the effect of time (i.e., the outcome trajectory over time) was different between conditions. We also used contrasts to test if the change from baseline to week 4 was 
different between conditions and reported the $95 \% \mathrm{Cl}$. For our safety and tolerability outcomes we compared the rates of serious adverse events and adverse events between the two conditions. Analysis was completed using SAS 9.3 (SAS Institute, Cary North Carolina, USA) and SPSS 23.0 (IBM Corporation, Armonk New York, USA) software. Study results are reported in accordance with the CONSORT extension for non-pharmacologic interventions [50] and the trial was registered with ClinicalTrials.gov, number NCT01860157.

\section{RESULTS}

Participant flow and sample characteristics

The flow of participants is presented in Fig. 1. Participants were recruited from June 2013 until July 2016 with final follow-up in November 2016. The intention-to-treat (ITT) sample was defined as all eligible participants randomized to $\mathrm{H} 1$ coil treatment and included 25 and 27 participants in active and sham rTMS, respectively. Trial recruitment was stopped before the target sample size was reached due to ending of the grant funding period.

Baseline participants' characteristics are summarized in Table 1: there were no differences between the two groups. Forty-seven participants $(90.4 \%)$ completed the acute course, and there were no baseline demographic or clinical differences between these 47 participants and the 5 who dropped out (who were all in the active condition).

\section{Assessment of blinding}

Participants were asked to guess their condition, and 17 of 25 participants $(68.0 \%)$ randomized to the active condition and 11 of 27 participants $(40.7 \%)$ randomized to the sham condition guessed correctly. The agreement between a participant's actual and perceived allocation suggested no agreement $(\mathrm{K}=0.09, p=$ 0.51) [51] indicating adequate participant blinding.

\section{Efficacy}

Primary outcome: remission rates. In the ITT sample, there was a significantly higher rate of remission in participants receiving active deep rTMS $(10 / 25,40.0 \% ; \mathrm{Cl}=21.1-61.3 \%)$ compared to sham rTMS $\left(4 / 27,14.8 \% ; \mathrm{Cl}=4.2-33.7 \% ; \mathrm{x}^{2}=4.2\right.$, d.f. $\left.=1, p<0.05\right)$ (Fig. 2). The NNT to achieve remission was $4.0(\mathrm{Cl}=2.1-56.5)$ and the RP of response was $2.7(\mathrm{Cl}=1.0-7.52)$. In the per protocol sample, defined as participants who completed 4 weeks of treatment, there was a significantly higher rate of remission in subjects receiving active deep rTMS $(n=10 ; 50.0 \%$; $\mathrm{Cl}=28.1-71.9 \%)$ compared to sham deep rTMS ( $n=4 ; 14.8 \%$; $\mathrm{Cl}=1.4-28.2 ; \mathrm{x}^{2}=6.8$, d.f. $\left.=1 ; p<0.05\right)$. In this sample, the NNT was $2.8(\mathrm{Cl}=1.6-10.5)$ and the $\mathrm{RP}$ was $3.4(\mathrm{Cl}=1.2-9.2)$. There were 14 participants who achieved remission by week 4 and received two additional weeks of treatment: 10 in the active treatment and 4 in sham treatment. The majority of patients remained in remission until week 6: 9/10 in the active treatment arm and $4 / 4$ in the sham treatment arm, which was not significantly different between groups (Fisher's exact $p=1.0$ ).

Secondary outcome: response rates. In the ITT sample, the rate of response was significantly higher with active deep rTMS (11/25; $44.0 \% ; \mathrm{Cl}=24.5-63.5 \%)$ than with sham $\mathrm{rTMS}(5 / 27 ; 18.5 \% ; \mathrm{Cl}=$ $3.9-33.2 \% ; \quad x^{2}=4.0$, d.f. $\left.=1, p<0.05\right)$. The NNT to achieve response was $3.9(\mathrm{Cl}=2.0-89.3)$ and the $\mathrm{RP}$ of response was 2.4 $(\mathrm{Cl}=1.0-5.9)$. In the per protocol sample, there was a significantly higher rate of response in subjects receiving active deep rTMS ( $n=11 ; 55.0 \% ; \mathrm{Cl}=33.2-76.8 \%)$ compared to sham deep rTMS $\left(n=5 ; 18.5 \% ; \mathrm{Cl}=3.9-33.2 \% ; \mathrm{x}^{2}=6.8\right.$, d.f. $\left.=1 ; p<0.05\right)$. In this sample, the NNT was $2.7(\mathrm{Cl}=1.6-9.8)$ and the RP was 3.0 $(\mathrm{Cl}=1.2-7.2)$. The 14 participants who received two additional weeks of treatment maintained their response out to week 6 in
Table 1. Participant demographic, clinical, and treatment characteristics

\begin{tabular}{|c|c|c|}
\hline & \multicolumn{2}{|c|}{ Group; mean \pm SD or no. (\%) } \\
\hline & $\begin{array}{l}\text { Active rTMS } \\
(n=25)\end{array}$ & $\begin{array}{l}\text { Sham rTMS } \\
(n=27)\end{array}$ \\
\hline \multicolumn{3}{|l|}{ Demographics } \\
\hline Age (years) [max-min] & $\begin{array}{l}65.0 \pm 5.5 \\
{[60-80]}\end{array}$ & $\begin{array}{l}65.4 \pm 5.5 \\
{[60-79]}\end{array}$ \\
\hline $\operatorname{Sex}(M / F)$ & $17: 8$ & $15: 12$ \\
\hline Education (years) & $17.0 \pm 2.5$ & $16.5 \pm 1.8$ \\
\hline Age at illness onset & $32.9 \pm 18.0$ & $30.4 \pm 18.6$ \\
\hline \multicolumn{3}{|l|}{ Clinical characteristics } \\
\hline Recurrent episode & $22(88.0)$ & $23(85.2)$ \\
\hline Duration of current episode (months) & $27.4 \pm 36.4$ & $43.1 \pm 65.2$ \\
\hline Number of episodes ${ }^{a}$ & $2.0 \pm 1.7$ & $3.3 \pm 2.8$ \\
\hline Comorbid psychiatric disorder ${ }^{b}$ & $4(16.7)$ & $8(29.6)$ \\
\hline Comorbid personality disorder ${ }^{b}$ & $0(0)$ & $1(3.7)$ \\
\hline Mean number of adequate trials ${ }^{c}$ & $1.6 \pm 1.0$ & $1.8 \pm 1.0$ \\
\hline Two or more failed medication trials & $10(40.0)$ & $15(55.5)$ \\
\hline Baseline HDRS-24 & $25.8 \pm 4.0$ & $27.6 \pm 4.1$ \\
\hline Baseline MMSE total & $29.2 \pm 0.9$ & $29.0 \pm 1.2$ \\
\hline \multicolumn{3}{|l|}{ Active medications } \\
\hline On any antidepressant ${ }^{d}$ & $18(72.0)$ & $17(63.0)$ \\
\hline Two antidepressants & $1(4.3)$ & $5(18.5)$ \\
\hline No antidepressants & $5(21.7)$ & $10(37.0)$ \\
\hline Benzodiazepine & $8(34.8)$ & $7(25.9)$ \\
\hline Atypical antipsychotic & $0(0)$ & $3(11.1)$ \\
\hline Antidepressant-antipsychotic combo & $0(0)$ & $3(11.1)$ \\
\hline Antidepressant and lithium & $1(4.3)$ & $1(3.7)$ \\
\hline \multicolumn{3}{|l|}{ rTMS characteristics } \\
\hline Number of rTMS treatments & $20.6 \pm 6.0$ & $20.8 \pm 2.2$ \\
\hline Average stimulus intensity ${ }^{e}$ & $118.4 \pm 0.01$ & $118.3 \pm 0.02$ \\
\hline Baseline motor threshold (\%) & $58.9 \pm 7.9$ & $59.1 \pm 13.6$ \\
\hline \multicolumn{3}{|c|}{$\begin{array}{l}\text { rTMS repetitive transcranial magnetic stimulation, SD standard deviation, } \\
\text { ATHF Antidepressant Treatment History Form, HDRS-24 } 24 \text {-item Hamilton } \\
\text { Depression Rating Scale, MMSE Mini-Mental Status Examination } \\
\text { a } N=9 \text { in the active group and } n=11 \text { in the sham group } \\
{ }^{b} N=24 \text { in the active group } \\
{ }^{c} \text { Adequate trial was defined as a trial of medication of sufficient dose and } \\
\text { duration to warrant an ATHF score of } \geq 3 \\
\text { d Any antidepressant whether dose was adequate or inadequate } \\
\text { eStimulus intensity titrated from } 100 \% \text { of resting motor threshold to } 120 \% \\
\text { by treatment five }\end{array}$} \\
\hline
\end{tabular}

the same proportions as remission: $9 / 10$ in the active treatment arm and 4/4 in the sham treatment arm (Fisher's exact $p=1.0$ ).

Secondary outcome: change in HDRS-24 score. From the mixedeffects model, the effect of time in both groups was characterized by a drop in HDRS-24 scores over time ( $F=36.5$, d.f. $=189.0 ; p<$ $0.001)$. There was no evidence for an effect of treatment condition $(F=3.3$, d.f. $=49.0 ; p=0.08)$. The time by treatment interaction was not significant $(F=0.9$, d.f. $=189.0 ; p=0.438$ ) (Supplemental Fig. 1).

Other secondary outcomes. From the mixed-effects model, the effect of time on the SSI, BSI, and SF-36 did not differ significantly between the active and sham rTMS conditions. Similarly, the changes of these measures from baseline to week 4 did not differ significantly (see Table 2). 
(A) $100 \%$ a Active rTMS $\square$ Sham rTMS

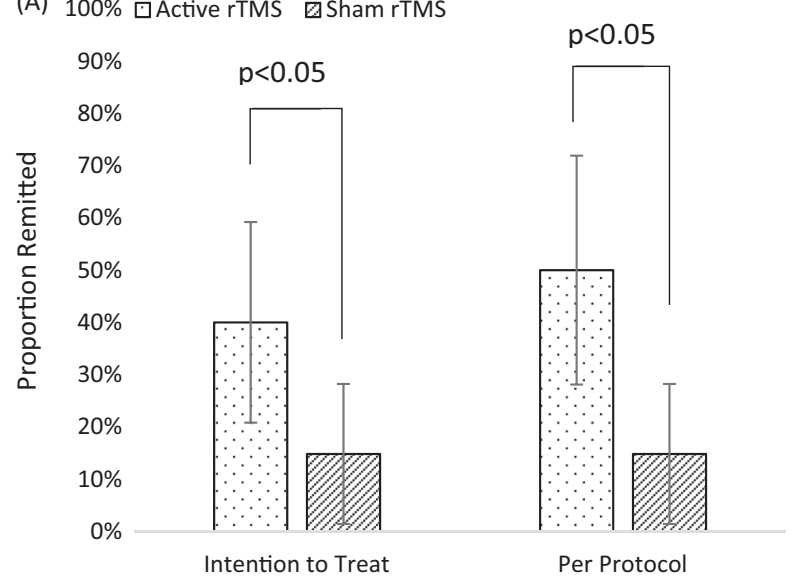

(B) $100 \%$ active rTMS $\square$ Sham rTMS

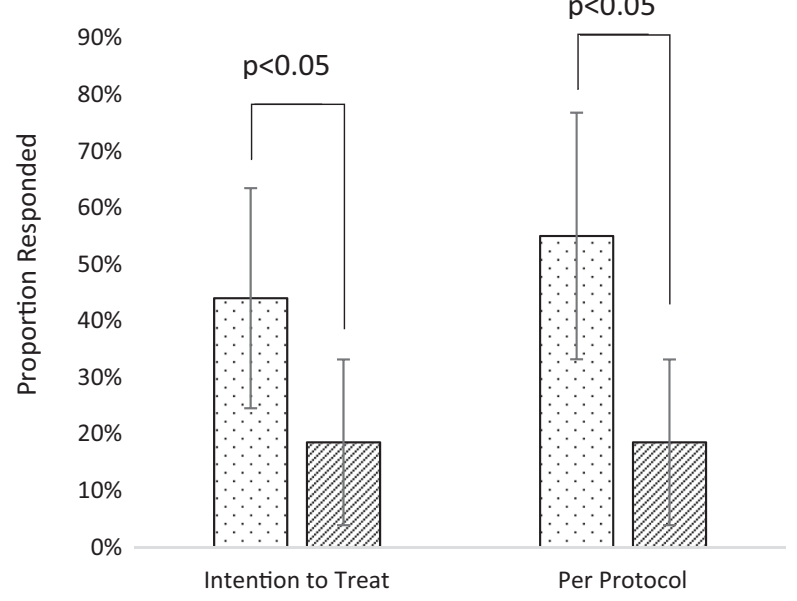

Fig. 2 a Remission and b response rates with 95\% confidence intervals based on 24-item Hamilton Depression Rating Scale between the intention-to-treat group (active $(n=25)$ and sham $(n=27))$ and per protocol group (active $(n=20)$ and sham $(n=27))$. In the primary trial outcome (remission in the intention-to-treat group) there were significantly more remitters who received active compared to sham rTMS $(p<0.05)$. rTMS repetitive transcranial magnetic stimulation
Change in cognitive function. From the mixed-effects model, there was a significant effect of time on the following RBANS scales: total scale $(F=37.1$, d.f. $=44.6 ; p<0.001)$, immediate memory scale $(F=12.5$, d.f. $=45.1 ; p<0.001)$, delayed memory scale $(F=45.8$, d.f. $=45.1 ; p<0.001)$, and language scale $(F=9.6$, d.f. $=47.3 ; p<0.003)$. There was also a significant effect of time on DKEFS-CWI (inhibition condition; $F=9.5$, d.f. $=45.7 ; p<0.003$ ). However, the effect of time did not differ significantly between the active and sham conditions on any cognitive (including executive) function measure (see Table 3 ).

Safety and tolerability

No serious adverse events were observed in this trial. Five of 52 participants in the ITT sample dropped out after a mean $( \pm S D)$ of $11.2 \pm 4.5$ sessions. All five were in the active condition; one participant did not wish to continue treatment despite symptom improvement; one due to worsening symptoms; one due to discomfort from stimulus; one required surgery for a corneal tear judged to be unrelated to treatment; and one had back pain and nausea secondary to renal colic judged to be unrelated to treatment. Adverse effects in the ITT sample $(n=52)$ are presented in Table 4 . The only adverse effect significantly more common in the active condition was pain $(16.0 \%$ vs $0 \%$, Fisher's exact $=0.05$ ).

\section{DISCUSSION}

To our knowledge, this is the first randomized controlled trial of extended duration deep rTMS in LLD. Our older participants randomized to active deep rTMS experienced a remission rate of $40.0 \%$ compared to $14.8 \%$ in the sham condition, corresponding to a NNT of 4. Similarly, deep rTMS produced a higher response rate. Overall tolerability of the $\mathrm{H} 1$ coil was good as only one participant discontinued treatment due to inability to tolerate the stimulus. Adverse effects were similar in the active and sham condition except for pain, which was more common with active deep rTMS.

In our trial, deep rTMS was associated with a meaningful remission rate $(40.0 \%)$ and a NNT smaller than typical NNTs of 5-10 reported in pharmacologic trials for older or younger persons with treatment-resistant depression [52-54]. Furthermore, while previous studies of conventional rTMS report lower remission rates in LLD than younger adults with MDD [9], the remission rate of active rTMS we found in this study $(40.0 \%)$ is

Table 2. Estimated marginal means from mixed effect model for symptom and quality of life assessments

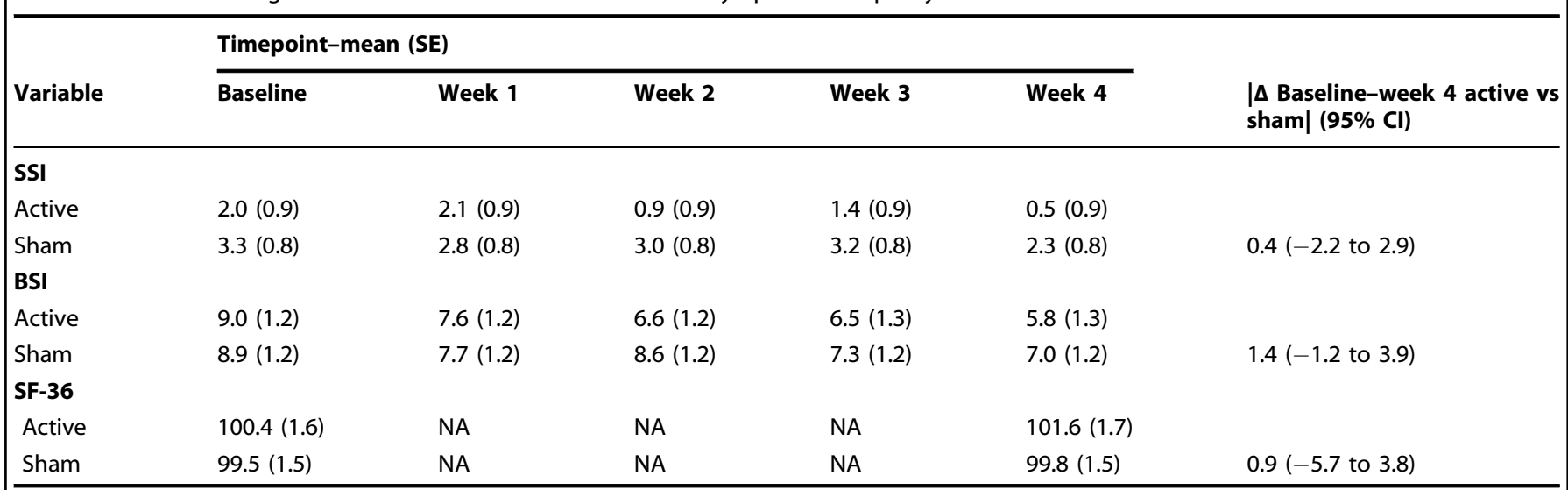

Fixed effects included in model were treatment allocation, time, and treatment $\times$ time interaction and participant identifiers were a random effect (random intercept)

SSI Scale for Suicidal Ideation, BSI Brief Symptom Inventory, SF-36 36-Item Short Form Survey 
Table 3. Estimated marginal means from mixed effect model for cognitive functioning assessments

\begin{tabular}{|c|c|c|c|}
\hline \multirow[b]{2}{*}{ Variable } & \multicolumn{2}{|c|}{$\begin{array}{l}\text { Timepoint-mean } \\
\text { (SE) }\end{array}$} & \multirow[b]{2}{*}{$\begin{array}{l}\mid \Delta \text { Baseline-week } 4 \text { active vs sham } \mid \\
(95 \% \mathrm{Cl})\end{array}$} \\
\hline & Baseline & Week 4 & \\
\hline \multicolumn{4}{|c|}{ RBANS-total ${ }^{a}$} \\
\hline Active & $-0.9(0.2)$ & $-0.4(0.2)$ & $0.0(-0.3$ to 0.3$)$ \\
\hline Sham & $-0.8(0.2)$ & $-0.3(0.2)$ & \\
\hline \multicolumn{4}{|c|}{ RBANS-immediate memory ${ }^{a}$} \\
\hline Active & $-0.8(0.3)$ & $-0.1(0.3)$ & $0.2(-0.8$ to 0.3$)$ \\
\hline Sham & $-0.7(0.2)$ & $-0.3(0.3)$ & \\
\hline \multicolumn{4}{|c|}{ RBANS-visuospatial $^{\mathrm{a}}$} \\
\hline Active & $-0.4(0.3)$ & $-0.1(0.3)$ & $0.3(-0.9$ to 0.2$)$ \\
\hline Sham & $0.3(0.3)$ & $0.3(0.3)$ & \\
\hline \multicolumn{4}{|c|}{ RBANS-language $^{a}$} \\
\hline Active & $-0.6(0.1)$ & $-0.4(0.1)$ & $0.2(-0.2$ to 0.7$)$ \\
\hline Sham & $-0.8(0.1)$ & $-0.4(0.1)$ & \\
\hline \multicolumn{4}{|c|}{ RBANS-attention $^{\mathrm{a}}$} \\
\hline Active & $-0.7(0.2)$ & $-0.5(0.2)$ & $0.2(-0.7$ to 0.2$)$ \\
\hline Sham & $-0.9(0.2)$ & $-0.9(0.2)$ & \\
\hline \multicolumn{4}{|c|}{ RBANS-delayed memory ${ }^{a}$} \\
\hline Active & $-0.8(0.2)$ & $-0.2(0.2)$ & $0.2(-0.2$ to 0.6$)$ \\
\hline Sham & $-0.7(0.2)$ & $0.1(0.2)$ & \\
\hline \multicolumn{4}{|c|}{ RBANS-codinga } \\
\hline Active & $-0.6(0.2)$ & $-0.5(0.2)$ & $0.1(-0.4$ to 0.2$)$ \\
\hline Sham & $-0.5(0.1)$ & $-0.4(0.2)$ & \\
\hline \multicolumn{4}{|c|}{ RBANS-semantic fluency } \\
\hline Active & $19.8(0.8)$ & $19.0(0.9)$ & $0.7(-1.8$ to 3.2$)$ \\
\hline Sham & $18.8(0.8)$ & $18.7(0.8)$ & \\
\hline \multicolumn{4}{|c|}{ DKEFS-CWI condition $3^{b}$} \\
\hline Active & $10.2(0.5)$ & $11.0(0.6)$ & $0.0(-1.1$ to 1.2$)$ \\
\hline Sham & $10.5(0.5)$ & $11.4(0.5)$ & \\
\hline \multicolumn{4}{|c|}{ DKEFS-CWI condition $4^{c}$} \\
\hline Active & $11.2(0.6)$ & $11.3(0.6)$ & $0.2(-1.6$ to 1.1$)$ \\
\hline Sham & $11.4(0.5)$ & $11.3(0.5)$ & \\
\hline \multicolumn{4}{|c|}{ DKEFS-TMT } \\
\hline Active & $8.6(0.7)$ & $9.0(0.8)$ & $0.1(-2.2$ to 2.0$)$ \\
\hline Sham & $9.4(0.7)$ & $9.7(0.7)$ & \\
\hline
\end{tabular}

Fixed effects included in model were treatment allocation, time, and treatment $\times$ time interaction and participant identifiers were a random effect (random intercept)

RBANS Repeatable Battery for the Assessment of Neuropsychological Status, DKEFS Delis-Kaplan Executive Function System, CWI Color Word Interference, TMT Trail Making Test, SE standard error, $\mathrm{Cl}$ confidence interval, $\Delta$ change in symptoms

${ }^{a} Z$-score calculated based on test mean and standard deviation to account for differences between test versions; ${ }^{2}$ Test score weighted based on inhibition; ${ }^{3}$ Test score weighted based on switching

bTest score weighted based on inhibition

${ }^{\mathrm{c}}$ Test score weighted based on switching

comparable to remission rates reported in the recent multicenter trial of deep rTMS in younger adults (32.6\%; NNT of 5.6) [20]. We also demonstrated durability of remission and response for up to 2 weeks after daily rTMS treatments. However, given the lack of an active comparator (i.e., standard rTMS coil), we were unable to determine if the superiority of active deep rTMS compared to
Table 4. Adverse effects by rTMS treatment condition

\begin{tabular}{lll}
\hline & \multicolumn{2}{l}{ Group; no. (\%) } \\
\cline { 2 - 3 } Adverse effect & Active $(\boldsymbol{n}=25)$ & Sham $(\boldsymbol{n}=27)$ \\
\hline Headache after treatment & $14(56.0)$ & $10(37.0)$ \\
Nasopharyngitis & $1(4.0)$ & $0(0)$ \\
Pain at stimulation site & $4(16.0)$ & $0(0)$ \\
Aphthous ulcer & $1(4.0)$ & $0(0)$ \\
Corneal abrasion & $1(4.0)$ & $0(0)$ \\
Dermatitis & $1(4.0)$ & $0(0)$ \\
Sinusitis & $1(4.0)$ & $0(0)$ \\
Nausea & $1(4.0)$ & $1(3.7)$ \\
Dental pain & $0(0)$ & $1(3.7)$ \\
Increased anxiety & $0(0)$ & $1(3.7)$ \\
\hline Bold adverse effects indicate $p<0.05$, Fisher's exact test \\
rTMS repetitive transcranial magnetic stimulation \\
\hline
\end{tabular}

sham was due to coil design features enabling the pulses to overcome age-related prefrontal cortical atrophy [55] or because the number of pulses per session in this trial (6012) was double the standard 3000 pulses per session $[27,28]$ and three times the number of pulses used in the multicenter $\mathrm{H} 1$ coil trial [20]. Irrespective of the underlying mechanism, our results suggest that LLD can be effectively treated with rTMS.

We also observed significant improvements over time in several of our secondary measures-i.e., the HDRS-24, BSI, and several cognitive functioning measures-but these improvements were independent of the conditions. This suggests a non-specific effect of participation in daily rTMS, which is congruent with the metaanalytic finding that sham rTMS is associated with large treatment effect sizes [56]. Given the brief duration of our trial (i.e., 4 weeks), it is unlikely that these improvements are due to the natural longitudinal course of depression [57]. While we did not observe treatment-attributable improvement in executive functioning within the short duration of our trial, this lack of difference between the active and sham conditions suggests that deep rTMS does not disturb cognitive functioning in older adults with LLD, which would be a significant advantage over ECT [6].

With respect to safety and tolerability, despite the age of our participants and the high doses of rTMS (6012 pulses per session at $120 \%$ RMT), deep rTMS was relatively well tolerated with only one dropout due to stimulus discomfort and the only adverse effect significantly more common in the active condition was pain. While this result compares favorably to prior trials $[27,28,58]$, future trials using the $\mathrm{H} 1$ coil will be needed to determine if the increased rate of pain causes more frequent dropouts and to compare tolerability with conventional rTMS coils.

\section{LIMITATIONS AND FUTURE WORK}

While the results of this study have important implications, some limitations need to be considered. First, we did not reach our target sample size and while the results of our primary analysis were statistically significant, the confidence intervals were large. Second, even though clinical evaluators, operators, and participants were blinded, adverse effects (specifically pain) were different between the two conditions. This has the potential to unblind allocation; however, previous rTMS studies using this and other devices have found that despite differing adverse effect rates, concealment of group allocation is maintained [20,27, 28]. Third, this trial assessed outcomes over a short period of time. Future work will need to determine the durability of response to rTMS given the chronic, recurrent course of LLD [59]. We were also 
unable to assess other potential longer-term effects of deep rTMS (e.g., on executive functions). Fourth, the higher dropout rate in active compared to sham deep rTMS was unexpected, and though only one dropout was due to tolerability, future studies will need to determine if this difference was the result of chance or reflects $\mathrm{H} 1$ coil tolerability. Finally, the high number of pulses delivered at each session required approximately $60 \mathrm{~min}$ and the length of these sessions may limit broader implementation of this approach.

\section{CONCLUSION}

This randomized controlled trial provides evidence for the efficacy and tolerability of high-dose deep rTMS for LLD. Participants who received active deep rTMS or sham rTMS had a remission rate of $40.0 \%$ and $14.8 \%$, respectively, yielding a low NNT of 4.0 . The $\mathrm{H} 1$ coil was well tolerated with only one participant dropping out due to inability to tolerate the stimulus, and pain was the only adverse effect more common with active rTMS. Based on these results, future studies with longer follow-up periods are justified to determine the role of deep rTMS for the treatment of LLD.

\section{ACKNOWLEDGEMENTS}

We thank Marcos Sanches for his assistance with the statistical analysis. This study was supported by the Canadian Institute for Health Research University-Industry Sponsored Operating Grant in conjunction with Brainsway Ltd. The funders of the study had no role in the study design, data collection, data analysis, data interpretation, or writing of the report. The authors attest that they had full access to all the data in the study and had final responsibility for the decision to submit the publication.

\section{ADDITIONAL INFORMATION}

Supplementary Information accompanies this paper at (https://doi.org/10.1038/ s41386-018-0121-x)

Competing interests: TSK receives research support from the University of Toronto and the Clinician Scientist Program through the Department of Psychiatry at the University of Toronto. In the last 3 years, ZJD has received research and equipment in-kind support for an investigator-initiated study through Brainsway Inc and Magventure Inc. His work was supported by the Ontario Mental Health Foundation (OMHF), the Canadian Institutes of Health Research (CIHR), the Brain and Behaviour Research Foundation, the National Institutes of Mental Health and the Temerty Family and Grant Family and through the Centre for Addiction and Mental Health (CAMH) Foundation and the Campbell Institute. YN receives research support from Otsuka Pharmaceutical Co., Ltd, Shionogi \& Co., Ltd, and Meiji Seika Pharma Co., Ltd. YN has also received research grants from Japan Health Foundation, Meiji Yasuda Mental Health Foundation, Mitsui Life Social Welfare Foundation, Takeda Science Foundation, and Daiichi Sankyo Scholarship Donation Program. YN has received equipment in-kind support for an investigator-initiated study from Magventure Inc. YK has no disclosures. JD receives research support from $\mathrm{CIHR}, \mathrm{NIH}$, Brain Canada Weston Brain Institute, and the Toronto General and Western Hospital Foundation, as well as in-kind (equipment) support from Magventure for an investigator-initiated study. TKR receives research support from the Weston Brain Institute, Canada Research Chair program, and the Canada Foundation for Innovation. YL has served as a consultant and has financial interests in Brainsway Ltd. AZ is a co-inventor of the TMS H-coils and serves as consultant for, and has financial interests in, Brainsway Ltd. He receives research support from the European Commission (Horizon 2020 program), $\mathrm{NIH}$, the Israel Science Foundation, the Israel Ministry of Health and equipment support from Brainsway Ltd. MAB receives research support from the US National Institutes of Health $(\mathrm{NIH})$. BHM currently receives research financial support from Brain Canada, the CAMH Foundation, the US Patient-Centered Outcomes Research Institute (PCORI), and the US National Institute of Health (NIH); and in-kind support from Capital Solution Design LLC (software used in a study founded by CAMH Foundation), Eli Lilly (medications for a NIH-funded clinical trial), Capital Solution Design LLC (software used in a study founded by CAMH Foundation), HAPPYneuron (software used in a study founded by Brain Canada), and Pfizer (medications for a NIH-funded clinical trial). Within the past 5 years, he has also received research support from the Canadian Institutes of Health Research (CIHR), Bristol-Myers Squibb (medications for a NIH-funded clinical trial), Pfizer (medications for a NIH-funded clinical trial), and Eli Lilly (medications for a NIH-funded clinical trial). He directly owns stock of General Electric (less than \$5000). DMB receives research support from the Canadian Institutes of Health Research (ClHR), Brain Canada, Weston Brain Institute, National Institutes of Health (NIH), Temerty Family through the Centre for Addiction and Mental Health (CAMH) Foundation, and the Campbell Family Research Institute. He received non-salary operating funds and in-kind equipment support from Brainsway Ltd for an investigator-initiated study. He is the site-principal investigator for three sponsor-initiated clinical trials from Brainsway Ltd. He received in-kind equipment support from Tonika/Magventure for an investigatorinitiated study. He received medication supplies from Indivior for an investigatorinitiated trial. He participated in one advisory board meeting for Janssen.

Publisher's note: Springer Nature remains neutral with regard to jurisdictional claims in published maps and institutional affiliations.

\section{REFERENCES}

1. World Health Organization. Depression and other common mental disorders: global health estimates. Geneva: World Health Organization; 2017.

2. World Health Organization. Mental health and older adults. Geneva: World Health Organization; 2016. http://www.who.int/mediacentre/factsheets/fs381/en/.

3. Mulsant BH, Pollock BG. Treatment-resistant depression in late life. J Geriatr Psychiatry Neurol. 1998;11:186-93.

4. Whyte EM, Basinski J, Farhi P, Dew MA, Begley A, Mulsant BH, et al. Geriatric depression treatment in nonresponders to selective serotonin reuptake inhibitors. J Clin Psychiatry. 2004;65:1634-41.

5. Gratzer D, Goldbloom D. Making evidence-based psychotherapy more accessible in Canada. Can J Psychiatry. 2016;61:618-23.

6. Prudic J. Strategies to minimize cognitive side effects with ECT: aspects of ECT technique. J ECT. 2008;24:46-51.

7. Slotema CW, Blom JD, Hoek HW, Sommer IEC. Should we expand the toolbox of psychiatric treatment methods to include Repetitive Transcranial Magnetic Stimulation (rTMS)? A meta-analysis of the efficacy of rTMS in psychiatric disorders. J Clin Psychiatry. 2010;71:873-84.

8. Brunoni AR, Chaimani A, Moffa AH, Razza LB, Gattaz WF, Daskalakis ZJ, et al. Repetitive transcranial magnetic stimulation for the acute treatment of major depressive episodes: a systematic review with network meta-analysis. JAMA Psychiatry. 2017;74:143-52.

9. Blumberger DM, Hsu JH, Daskalakis ZJ. A review of brain stimulation treatments for late-life depression. Curr Treat Options Psychiatry. 2015;2:413-21.

10. Fregni F, Marcolin MA, Myczkowski M, Amiaz R, Hasey G, Rumi DO, et al. Predictors of antidepressant response in clinical trials of transcranial magnetic stimulation. Int J Neuropsychopharmacol. 2006;9:641.

11. Manes F, Jorge R, Morcuende M, Yamada T, Paradiso S, Robinson RG. A controlled study of repetitive transcranial magnetic stimulation as a treatment of depression in the elderly. Int Psychogeriatr. 2001;13:225-31.

12. Mosimann UP, Marré SC, Werlen S, Schmitt W, Hess CW, Fisch HU, et al. Antidepressant effects of repetitive transcranial magnetic stimulation in the elderly: correlation between effect size and coil-cortex distance. Arch Gen Psychiatry. 2002;59:560-1.

13. Deng Z-D, Lisanby SH, Peterchev AV. Electric field depth-focality tradeoff in transcranial magnetic stimulation: simulation comparison of 50 coil designs. Brain Stimul. 2013;6:1-13.

14. Roth Y, Amir A, Levkovitz Y, Zangen A. Three-dimensional distribution of the electric field induced in the brain by transcranial magnetic stimulation using figure-8 and deep H-coils. J Clin Neurophysiol. 2007;24:31-38.

15. Roth Y, Pell GS, Chistyakov AV, Sinai A, Zangen A, Zaaroor M. Motor cortex activation by $\mathrm{H}$-coil and figure- 8 coil at different depths. Combined motor threshold and electric field distribution study. Clin Neurophysiol. 2014;125:336-43.

16. Roth Y, Zangen A, Hallett M. A coil design for transcranial magnetic stimulation of deep brain regions. J Clin Neurophysiol. 2002;19:361-70.

17. Zangen A, Roth $Y$, Voller B, Hallett M. Transcranial magnetic stimulation of deep brain regions: evidence for efficacy of the H-Coil. Clin Neurophysiol. 2005;116:775-9.

18. Berlim MT, Eynde F, Van den, Tovar-Perdomo S, Chachamovich E, Zangen A, Turecki G. Augmenting antidepressants with deep transcranial magnetic stimulation (DTMS) in treatment-resistant major depression. World J Biol Psychiatry. 2014;15:570-8.

19. Isserles $M$, Rosenberg O, Dannon P, Levkovitz $Y$, Kotler $M$, Deutsch $F$, et al. Cognitive-emotional reactivation during deep transcranial magnetic stimulation over the prefrontal cortex of depressive patients affects antidepressant outcome. J Affect Disord. 2011;128:235-42.

20. Levkovitz Y, Isserles M, Padberg F, Lisanby SH, Bystritsky A, Xia G, et al. Efficacy and safety of deep transcranial magnetic stimulation for major depression: a prospective multicenter randomized controlled trial. World Psychiatry. 2015;14:64-73. 
21. Cullen KR, Jasberg S, Nelson B, Klimes-Dougan B, Lim KO, Croarkin PE. Seizure induced by deep transcranial magnetic stimulation in an adolescent with depression. J Child Adolesc Psychopharmacol. 2016;26:637-41.

22. Harel EV, Zangen A, Roth Y, Reti I, Braw Y, Levkovitz Y. H-coil repetitive transcranial magnetic stimulation for the treatment of bipolar depression: an add-on, safety and feasibility study. World J Biol Psychiatry. 2011; 12:119-26.

23. Levkovitz Y, Rabany L, Harel EV, Zangen A. Deep transcranial magnetic stimulation add-on for treatment of negative symptoms and cognitive deficits of schizophrenia: a feasibility study. Int J Neuropsychopharmacol. 2011;14:991-6.

24. Rosenberg O, Isserles M, Levkovitz Y, Kotler M, Zangen A, Dannon PN. Effectiveness of a second deep TMS in depression: a brief report. Prog Neuropsychopharmacol Biol Psychiatry. 2011;35:1041-4.

25. Nahas Z, Li X, Kozel FA, Mirzki D, Memon M, Miller K, et al. Safety and benefits of distance-adjusted prefrontal transcranial magnetic stimulation in depressed patients 55-75 years of age: a pilot study. Depress Anxiety. 2004;19:249-56

26. Mosimann UP, Schmitt W, Greenberg BD, Kosel M, Müri RM, Berkhoff M, et al. Repetitive transcranial magnetic stimulation: a putative add-on treatment for major depression in elderly patients. Psychiatry Res. 2004;126:123-33.

27. George MS, Lisanby SH, Avery D, McDonald WM, Durkalski V, Pavlicova M, et al. Daily left prefrontal transcranial magnetic stimulation therapy for major depressive disorder. Arch Gen Psychiatry. 2010;67:507.

28. O'Reardon JP, Solvason HB, Janicak PG, Sampson S, Isenberg KE, Nahas Z, et al. Efficacy and safety of transcranial magnetic stimulation in the acute treatment of major depression: a multisite randomized controlled trial. Biol Psychiatry. 2007;62:1208-16.

29. Sun Y, Farzan F, Mulsant BH, Rajji TK, Fitzgerald PB, Barr MS, et al. Indicators for remission of suicidal ideation following magnetic seizure therapy in patients with treatment-resistant depression. JAMA Psychiatry. 2016;73:337-45.

30. Voineskos D, Levinson AJ, Sun Y, Barr MS, Farzan F, Rajji TK, et al. The relationship between cortical inhibition and electroconvulsive therapy in the treatment of major depressive disorder. Sci Rep. 2016;6:37461.

31. Jesus DR, de, Favalli GP, de S, Hoppenbrouwers SS, Barr MS, Chen R, Fitzgerald PB, et al. Determining optimal rTMS parameters through changes in cortical inhibition. Clin Neurophysiol. 2014;125:755-62.

32. Holtzheimer PE, McDonald WM, Mufti M, Kelley ME, Quinn S, Corso G, et al. Accelerated repetitive transcranial magnetic stimulation for treatment-resistant depression. Depress Anxiety. 2010;27:960-3.

33. Koenig AM, DeLozier IJ, Zmuda MD, Marron MM, Begley AE, Anderson SJ, et al. Neuropsychological functioning in the acute and remitted States of late-life depression. J Alzheimers Dis. 2015;45:175-85.

34. Butters MA, Whyte EM, Nebes RD, Begley AE, Dew MA, Mulsant BH, et al. The nature and determinants of neuropsychological functioning in late-life depression. Arch Gen Psychiatry. 2004;61:587-95.

35. Martis B, Alam D, Dowd SM, Hill SK, Sharma RP, Rosen C, et al. Neurocognitive effects of repetitive transcranial magnetic stimulation in severe major depression. Clin Neurophysiol. 2003;114:1125-32.

36. Ilieva IP, Alexopoulos GS, Dubin MJ, Morimoto SS, Victoria LW, Gunning FM. Agerelated repetitive transcranial magnetic stimulation effects on executive function in depression: a systematic review. Am J Geriatr Psychiatry.2018;26:334-46.

37. First MB, Spitzer RL, Gibbon M, Williams JBW. Structured Clinical Interview for DSM-IV-TR Axis I Disorders, research version, patient edition. New York: Biometrics Research; 2002.

38. Hamilton M. A rating scale for depression. J Neurol Neurosurgry Psychiatry. 1960;23:56-62.

39. Sackeim HA, Prudic J, Devanand DP, Decina P, Kerr B, Malitz S. The impact of medication resistance and continuation pharmacotherapy on relapse following response to electroconvulsive therapy in major depression. J Clin Psychopharmacol. 1990;10:96-104.
40. Fava M, Rush AJ, Thase ME, Clayton A, Stahl SM, Pradko JF, et al. 15 years of clinical experience with bupropion $\mathrm{HCl}$ : from bupropion to bupropion $\mathrm{SR}$ to bupropion XL. Prim Care Companion J Clin Psychiatry. 2005;7:106-13.

41. Ziemann U, Lönnecker S, Steinhoff BJ, Paulus W. The effect of lorazepam on the motor cortical excitability in man. Exp Brain Res. 1996;109:127-35.

42. Ziemann U, Lönnecker S, Steinhoff BJ, Paulus W. Effects of antiepileptic drugs on motor cortex excitability in humans: a transcranial magnetic stimulation study. Ann Neurol. 1996:40:367-78.

43. Levkovitz Y, Harel EV, Roth Y, Braw Y, Most D, Katz LN, et al. Deep transcranial magnetic stimulation over the prefrontal cortex: evaluation of antidepressant and cognitive effects in depressive patients. Brain Stimul. 2009;2:188-200.

44. Rossini PM, Barker AT, Berardelli A, Caramia MD, Caruso G, Cracco RQ, et al. Noninvasive electrical and magnetic stimulation of the brain, spinal cord and roots: basic principles and procedures for routine clinical application. Report of an IFCN committee. Electroencephalogr Clin Neurophysiol. 1994;91:79-92.

45. Beck AT, Kovacs M, Weissman A. Assessment of suicidal intention: the Scale for Suicide Ideation. J Consult Clin Psychol. 1979;47:343-52.

46. Dombrovski AY, Mulsant BH, Houck PR, Mazumdar S, Lenze EJ, Andreescu C, et al. Residual symptoms and recurrence during maintenance treatment of late-life depression. J Affect Disord. 2007;103:77-82.

47. Derogatis LR, Melisaratos N. The Brief Symptom Inventory: an introductory report. Psychol Med. 1983;13:595-605.

48. Randolph C, Tierney MC, Mohr E, Chase TN. The Repeatable Battery for the Assessment of Neuropsychological Status (RBANS): preliminary clinical validity. J Clin Exp Neuropsychol. 1998;20:310-9.

49. Delis DC, Kramer JH, Kaplan E, Holdnack J. Reliability and validity of the DelisKaplan Executive Function System: an update. J Int Neuropsychol Soc. 2004;10:301-3.

50. Boutron I, Altman DG, Moher D, Schulz KF, Ravaud P, CONSORT NPT Group. CONSORT Statement for Randomized Trials of Nonpharmacologic Treatments: A 2017 Update and a CONSORT Extension for Nonpharmacologic Trial Abstracts. Ann Intern Med. 2017;167:40

51. McHugh ML. Interrater reliability: the kappa statistic. Biochem Med. 2012;22:276-82.

52. Crossley NA, Bauer M. Acceleration and augmentation of antidepressants with lithium for depressive disorders: two meta-analyses of randomized, placebocontrolled trials. J Clin Psychiatry. 2007;68:935-40.

53. Lenze EJ, Mulsant BH, Blumberger DM, Karp JF, Newcomer JW, Anderson SJ, et al. Efficacy, safety, and tolerability of augmentation pharmacotherapy with aripiprazole for treatment-resistant depression in late life: a randomised, doubleblind, placebo-controlled trial. Lancet. 2015;386:2404-12.

54. Nelson JC, Papakostas Gl. Atypical antipsychotic augmentation in major depressive disorder: a meta-analysis of placebo-controlled randomized trials. Am J Psychiatry. 2009;166:980-91.

55. Kozel FA, Nahas Z, deBrux C, Molloy M, Lorberbaum JP, Bohning D, et al. How coil-cortex distance relates to age, motor threshold, and antidepressant response to repetitive transcranial magnetic stimulation. J Neuropsychiatry Clin Neurosci. 2000;12:376-84.

56. Brunoni AR, Lopes M, Kaptchuk TJ, Fregni F, Saikali K. Placebo response of nonpharmacological and pharmacological trials in major depression: a systematic review and meta-analysis. PLoS One. 2009;4:e4824.

57. Richards D. Prevalence and clinical course of depression: A review. Clin Psychol Rev. 2011:31:1117-25.

58. Blumberger DM, Maller JJ, Thomson L, Mulsant BH, Rajji TK, Maher M, et al. Unilateral and bilateral MRI-targeted repetitive transcranial magnetic stimulation for treatment-resistant depression: a randomized controlled study. J Psychiatry Neurosci. 2016:41:E58-E66.

59. Comijs HC, Nieuwesteeg J, Kok R, Marwijk HW, van, Mast RC, van der, Naarding P, et al. The two-year course of late-life depression; results from the Netherlands study of depression in older persons. BMC Psychiatry. 2015;15:20. 